\title{
Evaluación del crecimiento de la microalga chlorella sorokiniana en diferentes medios de cultivo en condiciones autotróficas y mixotróficas
}

\author{
Evaluating microalgae Chlorella sorokiniana growth in different \\ culture mediums in autotrophic and mixotrophic conditions
}

\section{Avaliação do crescimento da Chlorella sorokiniana em diferentes meios de cultura sob condições autotróficas e mixotróficas} \author{
Jorge Padilla ${ }^{4}$, Angélica M. Otero-Paternina ${ }^{5}$

\footnotetext{
Bióloga, Msc, Facultad de Ciencias Básicas e Ingenierías,
} \\ 2 Química, Esp. MSc (C), Facultad de Ciencias Básicas e Ingenierías. \\ 3,4 Estudiantes de Ingeniería Agronómica, \\ 5 Profesional en acuicultura, Msc (C), Grupo Gritox \\ Universidad de los Llanos. Email: mlortizm@unillanos.edu.co
}

Martha L. Ortiz-Moreno ${ }^{1}$, Caroll E. Cortés - Castillo², Julián Sánchez-Villarraga ${ }^{3}$,

Recibido: noviembre 22 de $2010 \quad$ Aceptado: diciembre 5 de 2011

\begin{abstract}
Resumen
Chlorella sorokiniana es una microalga con alto potencial biotecnológico por su capacidad de sintetizar ácidos grasos de interés industrial, rápido crecimiento y capacidad de adaptación a diferentes fuentes de nutrientes tanto en regímenes autotróficos como mixotroficos e inclusive heterotróficos. El objetivo de este trabajo fue identificar el medio de cultivo que permitiera un máximo crecimiento de C. sorokiniana para su producción masiva. Se evaluaron los medios de cultivo Sueoka, Guillard y Remital (fertilizante NPK: 1, 2, 3, 4, 5g/L), que ofrecen nutrientes en condiciones autotróficas y lixiviado de gallinaza en concentraciones del 30, 50 y 80\%, en régimen mixotrófico. Se registró una densidad celular máxima en medio Remital de 86,5 0,75 x $10^{7}$ células/ml y una velocidad específica de crecimiento media de 0,3 generaciones/ día. Los test estadísticos de Tukey y Fisher indicaron que el Remital fue el mejor medio de cultivo y las condiciones autotróficas fueron ideales para el crecimiento de C. sorokiniana. El uso de altas concentraciones de Remital no mejoro significativamente el crecimiento de la microalga. Por tanto, se recomienda el uso de Remital en bajas concentraciones (1g/L) como la mejor opción para la producción masiva de biomasa de C. sorokiniana.
\end{abstract}

Palabras claves: Chlorella sorokiniana, densidad celular, lixiviado de gallinaza, mixotrofia, autotrofia.

\begin{abstract}
Chlorella sorokiniana is a microalgae having great biotechnological potential due to its ability to synthesise fatty acids having industrial interest, rapid growth and the ability to adapt to differing nutrient sources in both autotrophic and mixotrophic regimens, and even heterotrophic ones. This work was aimed at identifying the culture medium which would lead
\end{abstract}


to maximum C. sorokiniana growth to enable its mass production. Sueoka, Guillard and Remital culture mediums (1, 2, $3,4,5 \mathrm{~g} / \mathrm{L}$ NPK fertiliser) were evaluated; they offer nutrients in autotrophic conditions and 30\%, 50\% and $80 \%$ concentration poultry manure leachate in a mixotrophic regimen. $86.5 \quad 0.75 \times 10^{7}$ cells $/ \mathrm{ml}$ maximum cell density and mean 0.3 generations/day specific growth rate were recorded in Remital medium. Tukey and Fisher statistical tests indicated that Remital was the best culture medium and that autotrophic conditions were ideal for $C$. sorokiniana growth. Using high concentrations of Remital did not significantly improve microalgae growth. Using low concentrations of Remital (1g/L) is thus recommended as the best option for mass production of $C$. sorokiniana biomass.

Key words: Chlorella sorokiniana, cell density, poultry manure leachate, mixotrophy, autotrophy.

\section{Resumo}

Chlorella sorokiniana é uma microalga com alto potencial biotecnológico por sua capacidade de sintetizar ácidos graxos de interesse industrial, rápido crescimento e capacidade de adaptação a diferentes fontes de nutrientes em regimes autotróficos, mixotróficos assim como também heterotróficos. O objetivo deste trabalho foi identificar o meio de cultura que favorece-se um máximo crescimento da C. sorokiniana para sua produção massiva. Avaliaram-se os meios de cultura Sueoka, Guillard e Remital (fertilizante NPK: 1, 2, 3, 4, 5g/L), que oferecem nutrientes em condições autotróficas e lixiviado de galinhaça em concentrações de 30, 50 e $80 \%$, em regime mixotrófico. Neste trabalho encontro-se uma densidade celular máxima em meio Remital de 86,5 0,75 x $10^{7}$ células $/ \mathrm{ml}$ e uma velocidade específica de crescimento meia de 0,3 gerações/dia. Os testes estatísticos de Tukey e Fisher mostraram que o Remital foi o melhor meio de cultura e as condições autotróficas foram ideais para o crescimento da C. sorokiniana. O uso de altas concentrações do Remital não melhoro significativamente o crescimento da microalga. Para tanto, recomenda-se o uso do Remital em baixas concentrações $(1 \mathrm{~g} / \mathrm{L})$ como a melhor opção para a produção massiva de biomassa da $C$. sorokiniana.

Palavras chave: Chlorella sorokiniana, densidade celular, lixiviado de galinhaça, mixotrofia, autotrofia.

\section{Introducción}

Las microalgas tienen la capacidad de crecer y hacer fotosíntesis con diferentes fuentes de nutrientes como las sales minerales, en condiciones autotroficas y sustancias orgánicas (como estiércoles y aguas residuales), en condiciones mixotroficas (Chinnasamy, 2010). Adicionalmente, algunas microalgas pueden crecer en condiciones heterotróficas, usando carbono orgánico en ausencia de luz (Xu et al., 2006). Esta plasticidad metabólica les permite adaptarse a diferentes ecosistemas y procesos biotecnológicos, generando biomasa que puede ser usada en la producción de alimentos, concentrados, compuestos bioactivos, biocombustibles, en la biorremediación y la producción de biofertilizantes (Chisti, 2007).

Una de las microalgas con mayor potencial biotecnológico es Chlorella sorokiniana (clorofícea) que se caracteriza por tener células esféricas $(5 \mathrm{~m}$ de diámetro), con un cloroplasto en forma de copa y un pirenoide. El género Chlorella es cosmopolita y por tanto se adapta a diversas condiciones ambientales y nutricionales (Wehr y Sheath, 2003), pudiendo ser encontrada en el fitoplancton de estanques y lagos, colonizando el suelo o como simbionte en protozoos ciliados. C. sorokiniana ha sido ampliamente estudiada con respecto a su fisiología, genética y por su producción de biomasa con alto contenido de lípidos, específicamente ácidos grasos insaturados omega 3, 6 y 9 , empleados en la obtención de suplementos nu- tricionales, cosméticos, farmaceúticos y biocombustibles (Ugwu et al., 2005; Spolaore et al., 2006; Sing y Goyal, 2007; Chisti, 2007).

Una de las limitantes principales del cultivo a escala industrial de $C$. sorokiniana son los costos de los componentes del medio de cultivo, que generalmente contiene fertilizantes inorgánicos derivados del petróleo (Chisti, 2007). El creciente aumento en los precios de los fertilizantes inorgánicos y su impacto ambiental negativo, han tenido como consecuencia la búsqueda de fuentes alternativas de nutrientes para los organismos fotosintéticos como las microalgas (Sevrin-Reyssac, 1998; Camargo y Alonso, 2006). Por tanto, la atención mundial se ha vuelto hacia el uso de materiales orgánicos de diversos orígenes como fertilizantes (Benedetti et al., 1998).

Los desechos animales, en su forma líquida, tienen una larga historia de uso como fuente de fósforo, nitrógeno y carbono para el crecimiento microalgal siendo de particular interés los desechos de la avicultura y porcicultura por sus grandes volúmenes y constante producción (Knud-Hansen, 1998; Venglovsky et al., 2006). Por ejemplo, la gallinaza es un desecho avícola que se compone de eyecciones de las aves de corral y del material usado como cama, que por lo general es cascarilla de arroz mezclada con cal. La gallinaza es un apreciado fertilizante orgánico, relativamente concentrado, de rápida acción y posee un alto contenido de urea (Hernandez y Cruz, 
1993; Lu et al., 2010). El Instituto Colombiano Agropecuario-ICA mediante resolución 150 de 2003 y la Norma Técnica Colombiana-NTC 5167 (ICONTEC, 2004), determina que todos los productores y comercializadores de gallinaza están obligados a estabilizar sanitariamente estos productos mediante compostaje y fermentación, dichos procesos pueden generar importantes cantidades de lixiviado, que sin un manejo adecuado se puede convertir en un problema ambiental generando contaminación en cuerpos de agua y malos olores. Una alternativa para el manejo del lixiviado de gallinaza es su uso como fuente de nutrientes para el crecimiento de microalgas (Rosales et al., 2007). Ya que las microalgas, en condiciones mixotróficas, pueden extraer fósforo y nitrógeno del lixiviado de la gallinaza disminuyendo su potencial eutrofizante de los cuerpos de agua y generando biomasa que puede ser utilizada en diferentes aplicaciones industriales (Pizarro et al., 2002; Chisti, 2007; Fernández y Benito, 2009).

El uso de estiércoles líquidos como fuente de nutrientes para el cultivo de algas ha sido evaluado ampliamente, pero la magnitud de sus efectos es variable, debido a que las propiedades fisicoquímicas de los estiércoles son heterogéneas y varían dependiendo de las condiciones fisiológicas de los animales fuente, de la calidad de su alimento y las condiciones climáticas (Cheeke et al., 1977; Groeneweg y Schluter, 1981; Travieso et al., 2006). Los estiércoles líquidos tienen como ventaja sus bajos costos y alta disponibilidad, pero requieren de un manejo adecuado para evitar procesos de descomposición bacteriana que generen productos tóxicos para las algas y riesgos sanitarios (Vásquez, 1994; Rosales et al., 2007; Kumar et al., 2010).

Una estrategia para el control de los productos tóxicos para las microalgas generados en la descomposición bacteriana de los estiércoles líquidos es su pretratamiento ("digestión") en condiciones anaeróbicas o aeróbicas. Estos pretratamientos permiten disminuir la demanda biológica de oxigeno (DBO) del estiércol líquido y su contenido de amonio, también estabilizan su $\mathrm{pH}$, siendo estas condiciones indispensables para el desarrollado adecuado de las microalgas (Costa et al., 2000; Olguín et al., 2001; Jiménez-Pérez et al., 2004; Kebede-Westhead et al., 2004; Wang et al., 2010). Específicamente, Rosales et al., (2007) recomiendan pretratar aeróbicamente la fracción soluble de gallinaza o lixiviado para viabilizar su uso en el cultivo de la microalga Chlorella sorokiniana.

En este contexto, es de suma importancia la realización de estudios sobre el crecimiento de $C$. sorokiniana en diferentes medios de cultivo para identificar las mejores condiciones nutricionales aplicables en la producción de biomasa microalgas con potencial biotecnológico. Por tal motivo, el objetivo de este trabajo fue evaluar el efecto del medio de cultivo en el crecimiento de la microalga $C$. sorokiniana en condiciones auto y mixotróficas.

\section{Materiales y métodos}

\section{Microalga}

Chlorella sorokiniana 211-32, donada por el Instituto de Bioquímica Vegetal y Fotosíntesis (IBVF) de la Universidad de Sevilla (España) mantenida en medio de cultivo Sueoka a 242 C con aireación y fotoperiodo de 12 horas con 2629306 lux.

\section{Medios de cultivo en condición mixotrófica: Preparación del lixiviado de gallinaza}

La gallinaza procedente de la granja avícola Bonanza ubicada en zona rural de la ciudad de Villavicencio (4 04'36,18" N 73 35'04,65"O; 380 msnm) fue tamizada $(0,5 \mathrm{~cm}$ ojo de malla) y se lavó con agua corriente en una proporción de $300 \mathrm{~g} /$ litro, el líquido resultante se dejó fermentar por 8 días en un recipiente abierto cubierto con una tela, esto se hizo para favorecer la degradación aeróbica de la gallinaza por parte de su microflora acompañante. El lixiviado obtenido se filtró con ayuda de una bomba de vacio con papel filtro Whatman \#1 de 0,45 m. El filtrado se dispuso en dosis de 30,50 y $80 \%$ en el medio de cultivo y se esterilizó en autoclave a $121 \mathrm{C}$ por 20 minutos (Rosales et al., 2007).

\section{Análisis bromatológico del lixiviado de gallinaza}

Este análisis fue desarrollado en el laboratorio de nutrición animal de la Universidad de los Llanos, aplicando los siguientes procedimientos: la preparación de muestras según la NTC4647 (ICONTEC, 1999a); materia seca por secado a $60 \mathrm{C}$ en horno de convección; cenizas según NTC4648 (ICONTEC, 2006), en la cual se toma $1 \mathrm{~g}$ de muestra y se lleva a $600 \mathrm{C}$ en una mufla por 3 horas; proteína cruda (NTC 4657 (ICONTEC, 1999b) por Microkjeldahl; extracto etéreo (NTC4969 (ICONTEC, 2001) por Soxleht con éter de petróleo por 1 hora; fibra cruda según NTC5122 (ICONTEC, 2002). Este análisis fue aplicado a una muestra de $1 \mathrm{~L}$ del lixiviado de gallinaza para conocer su contenido de nutrientes.

\section{Análisis fisicoquímico del lixiviado de gallinaza}

Este análisis fue desarrollado en el laboratorio de aguas de la Universidad de los Llanos, evaluando los 
siguientes parámetros: $\mathrm{pH}$, sólidos totales, demanda química de oxigeno (DQO), demanda biológica de oxigeno (DBO), ácidez, alcalinidad, turbidez, potasio, sodio, dureza cálcica y total, hierro, carbonatos, bicarbonatos y cloruros según las metodologías establecidas en la GTC2 (ICONTEC, 1994). Este análisis fue aplicado a una muestra de $1 \mathrm{~L}$ del lixiviado de gallinaza para determinar sus propiedades fisicoquímicas.

\section{Medios de cultivo en condición autrófica}

El medio Suoeka se compone de los siguientes elementos: $\mathrm{KH}_{2} \mathrm{PO}_{4} 8,3 \mathrm{Mm} ; \mathrm{K}_{2} \mathrm{HPO}_{4} 5,3 \mathrm{Mm} ; \mathrm{MgSO}_{4} .7 \mathrm{H}_{2} \mathrm{O}$ $0,25 \mathrm{Mm} ; \mathrm{CaCL}_{2} .2 \mathrm{H}_{2} \mathrm{O} 0,133 \mathrm{Mm}$; Trazas Hutner 1x; $\mathrm{NH}_{4} \mathrm{Cl}$ 9,35Mm. Trazas Hutner 200x: 12,7g de EDTA$\mathrm{Na} .2 \mathrm{H}_{2} \mathrm{O}$ en $250 \mathrm{ml}$ de agua, se disuelven uno a uno en su orden los siguientes reactivos en $500 \mathrm{ml}$ de agua a $100 \mathrm{C}: 2,28 \mathrm{~g}$ de $\mathrm{H}_{3} \mathrm{BO}_{3} ; 4,40 \mathrm{~g}$ de $\mathrm{ZnSO}_{4} .7 \mathrm{H}_{2} \mathrm{O} ; 1,02 \mathrm{~g}$ de $\mathrm{MnCl}_{2} .4 \mathrm{H}_{2} \mathrm{O} ; 1,00 \mathrm{~g}$ de $\mathrm{FeSO}_{4} .7 \mathrm{H}_{2} \mathrm{O} ; 0,32 \mathrm{~g}$ de $\mathrm{CoCl}_{2} .6 \mathrm{H}_{2} \mathrm{O} ; 0,32 \mathrm{~g}$ de $\mathrm{CuSO}_{4} .5 \mathrm{H}_{2} \mathrm{O} ; 0,22 \mathrm{~g}$ de $\left(\mathrm{NH}_{4}\right)$ $\mathrm{MoO}_{24} 4 \mathrm{H}_{2} \mathrm{O}$. Se mezclan las soluciones anteriores, se lleva a $100 \mathrm{C}$ y se ajusta el $\mathrm{pH}$ a $6,8 \mathrm{con} \mathrm{KOH}$ al $20 \%$. (a una temperatura inferior a 70 C) (IBVF, 2011).

El medio f/2 de Guillard contiene: $1 \mathrm{ml}$ de solución que contiene $0,08 \mathrm{~g}$ de $\mathrm{CoCl}_{2} \cdot 6 \mathrm{H}_{2} \mathrm{O}$ y $0,8 \mathrm{~g}$ de $\mathrm{CuSO}_{4} .5 \mathrm{H}_{2} \mathrm{O}$ en $100 \mathrm{ml}$ de agua destilada; que se agrega a una solución de $800 \mathrm{ml}$ con: $0,2 \mathrm{~g}$ de $\mathrm{FeCl}_{3} .6 \mathrm{H}_{2} \mathrm{O}, 0,06 \mathrm{~g}$ de $\mathrm{ZnSO}_{4} .7 \mathrm{H}_{2} \mathrm{O}, 0,12 \mathrm{~g}$ de $\mathrm{MnSO}_{4} \cdot \mathrm{H}_{2} \mathrm{O}, 0,03 \mathrm{~g}$ de $\mathrm{Na}_{2} \mathrm{MoO}_{4} \cdot 2 \mathrm{H}_{2} \mathrm{O} ; 1 \mathrm{ml}$ de solución de 1,2g de EDTA-Na en $900 \mathrm{ml}$ de agua destilada. Se ajusta el $\mathrm{pH}$ a 7,0 y añadir $10 \mathrm{~g} \mathrm{KNO}_{3}$ y $1,4 \mathrm{~g}$ de $\mathrm{KH}_{2} \mathrm{PO}_{4}$. Se lleva a volumen final de 1 litro y se lleva a 100 C (IBVF, 2011).

El medio Remital se preparó con 1g/L de abono Remital-M de la empresa Abocol que contiene $17 \%$ de nitrógeno total, fósforo asimilable $6 \%$, potasio soluble en agua $18 \%$, magnesio $2 \%$, azufre total $1,6 \%$, boro $0,2 \%$ y $0,1 \%$ de zinc. Este medio de cultivo fue efectivo para la proliferación de Chlorella vulgaris (Muñoz, 2009). Durante el desarrollo del presente trabajo también se analizó el crecimiento de Chlorella sorokiniana en las siguientes concentraciones $(1.0,2.0,3.0,4.0$ y $5.0 \mathrm{~g} / \mathrm{L})$, con el objetivo de encontrar aumentos en los parámetros de crecimiento de la microalga.

Los cultivos se realizaron por quintuplicado iniciando con una densidad celular de $1 \times 10^{7} \mathrm{cel} / \mathrm{ml}$ en erlenmeyers de $350 \mathrm{ml}$ con $200 \mathrm{ml}$ de medio de cultivo. Estos experimentos se realizaron con una intensidad luminosa de $2629 \pm 306$ lux en fotoperiodo de 12 horas, aireación constante y temperatura de $24 \pm 2 \mathrm{C}$ durante 15 días.

\section{Análisis de densidad celular}

La densidad celular fue determinada en todos los experimentos, por recuento en microscopio cada tres días hasta alcanzar la fase estacionaria, usando un hematocitómetro Neubauer. Los parámetros de cinética de crecimiento se calcularon a partir de los datos de densidad celular en fase exponencial, usando las formulas propuestas por Lobban et al., (1988).

\section{Análisis estadístico}

Los datos se analizaron mediante análisis de varianza (ANOVA) con un $\alpha=0,05$, para la determinación de tratamientos significativamente diferentes. En todos los casos en que la prueba $\mathrm{F}$ resultó significativa, se emplearon análisis de comparaciones múltiples (Test de Tukey y Fisher, Hsu y Dunnett, según el caso) para identificar el mejor tratamiento, con un nivel de significancia del 95\%, mediante el programa Minitab 14.

\section{Resultados}

\section{Características del lixiviado de gallinaza}

El análisis bromatológico del lixiviado de gallinaza mostró que esta fuente de nutrientes aporta $18 \%$ de nitrógeno en forma de proteína y $35 \%$ de minerales en la forma de cenizas (Tabla 1).

Tabla 1. Análisis bromatológico del lixiviado de gallinaza

\begin{tabular}{l|r}
\multicolumn{1}{c|}{ Parámetro } & \multicolumn{1}{c}{$\%$} \\
\hline Humedad final & 3,87 \\
Cenizas & 35,05 \\
Lípidos & 2,36 \\
Proteína & 17,97 \\
Carbohidratos & 38,66 \\
Fibra & 2,09 \\
\hline
\end{tabular}

El análisis fisicoquímico del lixiviado de gallinaza mostró $\mathrm{pH}$ ligeramente ácido $(6,7$ unidades de $\mathrm{pH})$, con un alto contenido de sólidos totales (1116 mg/L) y alta turbidez (5700 NTU). La demanda química de oxígeno-DQO fue de $6800 \mathrm{mg} / \mathrm{L}$ y la demanda biológica de oxígeno-DBO de $340 \mathrm{mg} / \mathrm{L}$ (Tabla 2). El lixiviado de gallinaza aporta $998 \mathrm{mg} / \mathrm{L}$ de potasio con respecto a los $180 \mathrm{mg} / \mathrm{L}$ que aporta el abono Remital. El nivel de nitrógeno que aportan el lixiviado de gallinaza y Remital es muy similar, $17,97 \%$ y $17 \%$, respectiva- 
mente. Estos datos indican que es un sustrato rico en nutrientes para el cultivo de microalgas

Tabla 2. Análisis fisicoquímico del lixiviado de gallinaza

\begin{tabular}{l|l}
\hline \multicolumn{1}{c|}{ Variable } & \multicolumn{1}{|c}{ Unidad de medida } \\
\hline $\mathrm{pH}$ & 6,70 \\
Sólidos totales & $1116 \mathrm{mg} / \mathrm{L}$ \\
$\mathrm{DQO}$ & $6800 \mathrm{mg} / \mathrm{L}$ \\
$\mathrm{DBO}$ & $340 \mathrm{mg} / \mathrm{L}$ \\
Acidez & $2774 \mathrm{mgCaCO} / \mathrm{L}$ \\
Alcalinidad & $2038 \mathrm{mgCaCO} / \mathrm{L}$ \\
Turbidez & $5700 \mathrm{NTU}$ \\
Potasio & $998 \mathrm{mg} / \mathrm{L}$ \\
Sodio & $140 \mathrm{mg} / \mathrm{L}$ \\
Dureza cálcica & $728 \mathrm{mg} / \mathrm{L}$ \\
Dureza total & $910 \mathrm{mg} / \mathrm{L}$ \\
Hierro & $20 \mathrm{mg} / \mathrm{L}$ \\
Carbonatos & $\mathrm{ND}$ \\
Bicarbonatos & $80 \mathrm{mg} \mathrm{CaCO} / \mathrm{L}$ \\
Cloruros & $583 \mathrm{mg} / \mathrm{L}$ \\
\hline
\end{tabular}

NTU: Unidades Nefelométricas de Turbidez; ND: No detectable

Comparación de medios de cultivo en condiciones auto y mixotróficas

\section{Comparación entre los medios de cultivo Sueoka, Guillard y el lixiviado de gallinaza}

En el primer ensayo se comparó los medios de cultivo Sueoka (medio de cultivo control), Guillard y el lixi- viado de gallinaza en las diferentes concentraciones (30, 50 y $80 \%$ ) encontrando diferencias significativas entre los medios de cultivo con un valor $\mathrm{P}=0,005$ en el ANOVA. Las pruebas de comparaciones múltiples de Dunnet y Hsu indican que el medio Guillard superó ampliamente las demás fuentes de nutrientes (Figura 1).

La densidad celular máxima para el medio Guillard fue de $13017,3 \times 10^{7}$ células $/ \mathrm{ml}$ con un tiempo de generación (g) de 1,5 días y una velocidad específica de crecimiento de 0,7 generaciones/día superando al medio Sueoka, al calcular los tiempos de generación para los medios de cultivo con lixiviado de gallinaza se encontró que fueron muy similares al medio de Guillard con valores que fluctuaron entre 1,3 a 1,8 días, siendo el menor tiempo para el lixiviado al $80 \%$. Los datos indican que el crecimiento en lixiviado presenta una fase de latencia más prolongada que en los medios inorgánicos (Tabla 3).

\section{Comparación entre los medios Remital, Guillard y lixiviado de gallinaza}

Luego de identificar los medios de cultivos más promisorios, se contrastaron con el medio Remital que fue reportado por Muñoz (2009) como la mejor fuente de nutrientes para el crecimiento de Chlorella vulgaris.

Con el medio de cultivo Remital, C. sorokiniana alcanzó una densidad celular mayor (86,5 0,75 x 10 células/ml) en comparación con los otros medios evaluados (Guillard y Lixiviado de gallinaza en sus diferentes concentraciones) (Figura 2).

EI ANOVA indica que hay diferencias significativas entre los medios de cultivo con un valor $\mathrm{P}=0,000$. Las pruebas de comparaciones múltiples de Tukey y

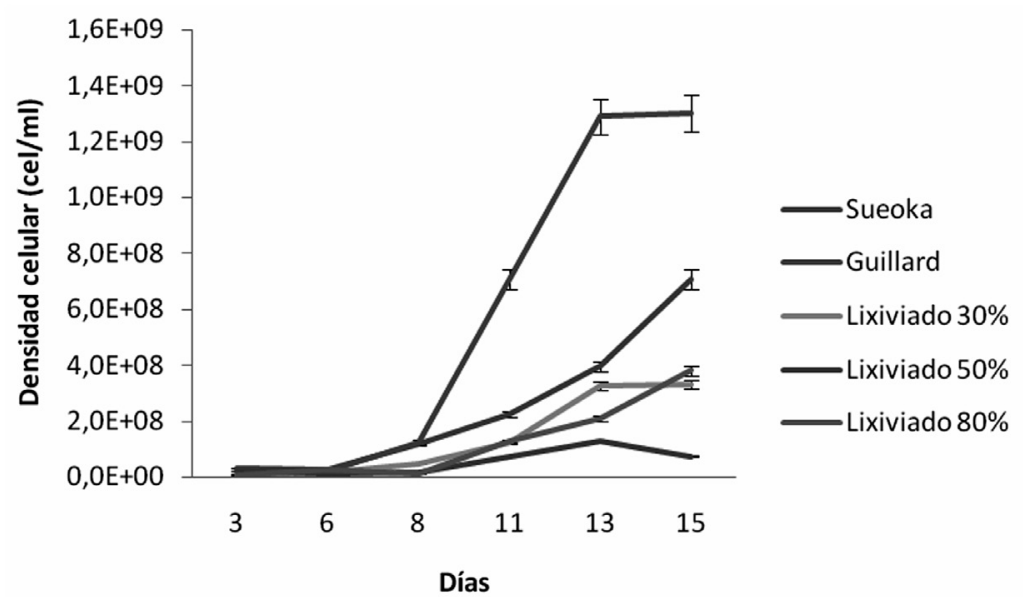

Figura 1. Comparación del efecto de los medios de cultivo sobre la densidad celular de C. sorokiniana. 
Tabla 3. Parámetros cinéticos de crecimiento de C. sorokiniana en diferentes medios de cultivo

\begin{tabular}{l|c|c|c|c|c}
\hline Medio de cultivo & $\begin{array}{c}\text { Densidad celular } \\
\text { final en la fase } \\
\text { exponencial (células/ } \\
\mathbf{m l}) \times \mathbf{1 0}^{\mathbf{7}}\end{array}$ & $\begin{array}{c}\text { Densidad celular } \\
\text { inicial en la fase } \\
\text { exponencial (células/ } \\
\mathbf{m l} \times \mathbf{1 \mathbf { 0 } ^ { 7 }}\end{array}$ & $\begin{array}{c}\mathrm{N} \\
\text { generaciones }\end{array}$ & $\begin{array}{c}\text { Tiempo de } \\
\text { generación } \\
\text { (días) }\end{array}$ & $\begin{array}{c}\text { Velocidad específica } \\
\text { de crecimiento } \\
\text { (generaciones.dia) }\end{array}$ \\
\hline Sueoka & $39 \pm 0,01$ & $12 \pm 2,6$ & 2 & 2,9 & 0,4 \\
Guillard & $130 \pm 17,3$ & $13 \pm 0,8$ & 3 & 1,5 & 0,7 \\
Lixiviado 30\% & $33 \pm 6,7$ & $4,9 \pm 0,2$ & 3 & 1,8 & 0,5 \\
Lixiviado 50\% & $13 \pm 1,11$ & $1,3 \pm 0,2$ & 3 & 1,5 & 0,6 \\
Lixiviado 80\% & $21 \pm 6,87$ & $1,5 \pm 0,4$ & 4 & 1,3 & 0,8 \\
\hline
\end{tabular}

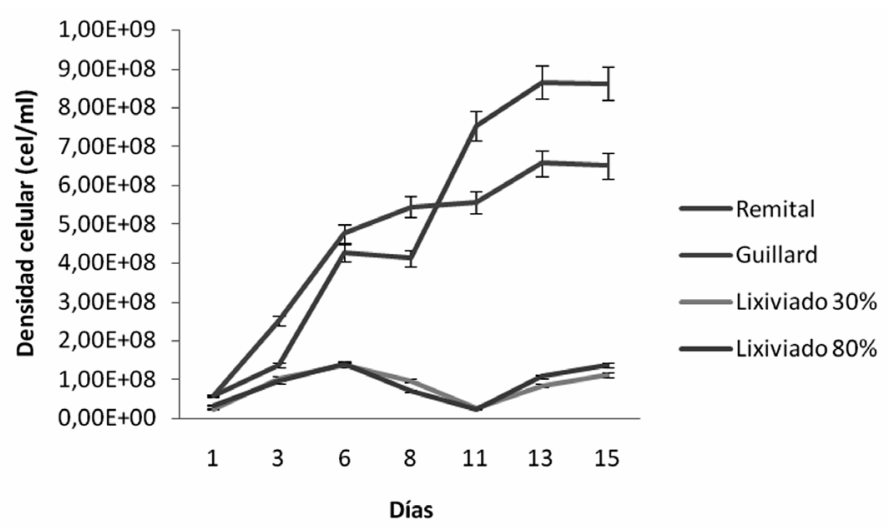

Figura 2. Comparación del efecto de los mejores medios de cultivo sobre la densidad celular de C. sorokiniana.

Fisher indican que el mejor medio de cultivo es Remital, seguido por Guillard (grupo de significancia B) y los medios con lixiviado de gallinaza tienen un comportamiento muy similar entre sí y propiciaron una menor densidad celular de $C$. sorokiniana (Grupo de significancia C) (Figura 2 y Tabla 4).

\section{Evaluación del efecto de la concentración del Remital en el crecimiento de C. sorokiniana}

Con el objetivo de encontrar la concentración de Remital que permitiera el mejor crecimiento de $C$. sorokiniana, se procedió a realizar ensayos referentes a la cinética de crecimiento de esta cepa en las siguientes concentraciones de Remital: 1.0, 2.0, 3.0, 4.0 y $5.0 \mathrm{~g} / \mathrm{L}$. En el experimento se encontró que a mayor concentración del Remital se presentaba una densidad celular mayor de la microalga, sin embargo, el ANOVA indico que no hay diferencias significativas entre los tratamientos ni en los parámetros cinéticos de la microalga, con un valor de $\mathrm{P}=0,123$ (Figura 3 y Tabla 5).

Tabla 4. Parámetros cinéticos de crecimiento de C. sorokiniana en los mejores medios de cultivo.

\begin{tabular}{l|c|c|c|c|c}
\hline Medio de cultivo & $\begin{array}{c}\text { Densidad celular } \\
\text { final en la fase } \\
\text { exponencial } \\
\text { (células/ml) } \times \mathbf{1 0}^{\mathbf{7}}\end{array}$ & $\begin{array}{c}\text { Densidad celular } \\
\text { inicial en la fase } \\
\text { exponencial } \\
\text { (células/ml) } \times 10^{7}\end{array}$ & $\begin{array}{c}\text { N } \\
\text { generaciones }\end{array}$ & $\begin{array}{c}\text { Tiempo de } \\
\text { generación } \\
\text { (días) }\end{array}$ & $\begin{array}{c}\text { Velocidad } \\
\text { específica de } \\
\text { crecimiento } \\
\text { (generaciones/día) }\end{array}$ \\
\hline Remital & $86,5 \pm 0,75$ & $5,76 \pm 0,24$ & 4 & 3,1 & 0,3 \\
Guillard & $65,7 \pm 0,80$ & $5,92 \pm 0,31$ & 3 & 3,5 & 0,3 \\
Lixiviado 30\% & $8,56 \pm 3,02$ & $2,40 \pm 0,64$ & 2 & 6,5 & 0,2 \\
Lixiviado 80\% & $10,7 \pm 3,15$ & $3,36 \pm 0,86$ & 2 & 7,2 & 0,1 \\
\hline
\end{tabular}




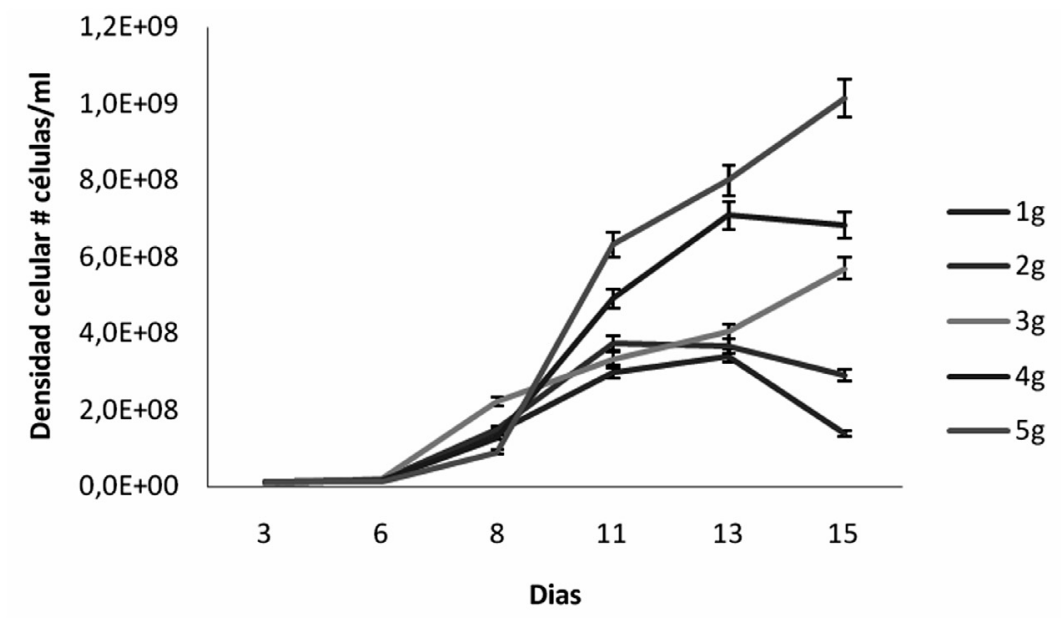

Figura 3. Efecto de la concentración de Remital (g/L) en el crecimiento de C. sorokiniana.

Tabla 5. Parámetros cinéticos de crecimiento de C. sorokiniana en diferentes concentraciones del medio Remital.

\begin{tabular}{c|c|c|c|c|c}
\hline $\begin{array}{c}\text { Concentración } \\
\text { de Remital } \\
\text { (g/L) }\end{array}$ & $\begin{array}{c}\text { Densidad celular } \\
\text { final en la fase } \\
\text { exponencial } \\
\text { (células/ml) } \times \mathbf{1 0}^{7}\end{array}$ & $\begin{array}{c}\text { Densidad celular } \\
\text { inicial en la fase } \\
\text { exponencial } \\
\text { (células/ml) } \times \mathbf{1 0}^{7}\end{array}$ & $\begin{array}{c}\text { N } \\
\text { generaciones }\end{array}$ & $\begin{array}{c}\text { Tiempo de } \\
\text { generación } \\
\text { (días) }\end{array}$ & $\begin{array}{c}\text { Velocidad } \\
\text { específica de } \\
\text { crecimiento } \\
\text { (generaciones/día) }\end{array}$ \\
\hline 1 & $34 \pm 3,7$ & $1,6 \pm 0,23$ & 4 & 1,6 & 0,6 \\
2 & $37 \pm 9,2$ & $1,7 \pm 0,13$ & 4 & 1,6 & 0,6 \\
3 & $40 \pm 3,0$ & $1,7 \pm 0,13$ & 5 & 1,5 & 0,7 \\
4 & $71 \pm 3,0$ & $1,7 \pm 0,35$ & 6 & 1,2 & 0,8 \\
\hline
\end{tabular}

\section{Discusion}

Chlorella es un género de microalgas clorofíceas que se caracteriza por presentar un rápido crecimiento en cultivo celular y por su capacidad de utilizar como sustrato nutricional compuestos orgánicos e inorgánicos (Wehr y Sheath, 2003; Xu et al., 2006; Chinnasamy, 2010). Por estos motivos, las especies del género Chlorella han sido ampliamente estudiadas para incluirlas en procesos biotecnológicos, que generen productos orientados a las industrias alimenticias farmaceúticas, de saneamiento ambiental, agropecuarias e energéticas (Chisti, 2007). Especificamente $C$. sorokiniana posee un gran potencial para la producción de ácidos grasos omega 3,6 y 9, además de la producción de biocombustibles (Ugwu et al., 2005; Spolaore et al., 2006; Sing y Goyal, 2007; Chisti, 2007).

En la producción industrial de biomasa de $C$. sorokiniana se requiere utilizar un medio de cultivo que ofrezca los nutrientes necesarios para el adecuado crecimiento del alga y de bajo costo. Los estiércoles líquidos como el lixiviado de gallinaza, constituyen una alternativa viable para reducir los costos en el cultivo de microalgas, pero estos sustratos deben ser pretratados adecuadamente para obtener los resultados deseados (Vásquez, 1994; Travieso et al., 2006; Rosales et al., 2007; Kumar et al., 2010).

El lixiviado de gallinaza pretratado aerobicamente usado en los experimentos mostró que es una fuente de proteínas y minerales de alta calidad, principalmente de potasio y nitrógeno, superando inclusive los niveles del primer nutriente en el fertilizante sintético Remital (Tabla 1 y 2). El lixiviado de gallinaza presento un $\mathrm{pH}$ ligeramente ácido $(\mathrm{pH}=6,7)$, con alto contenido de sólidos totales $(1116 \mathrm{mg} / \mathrm{L})$ y alta turbidez (5700 NTU). La demanda química de oxígenoDQO fue de $6800 \mathrm{mg} / \mathrm{L}$ superando ampliamente a la demanda biológica de oxígeno-DBO de $340 \mathrm{mg} / \mathrm{L}$, lo cual indica una degradación casi completa de los sustratos susceptibles de fermentación por microorganismos, esto muestra que la maduración previa al cultivo 
algal del lixiviado de gallinaza fue efectiva para permitir la mineralización de los nutrientes utilizables en la fotosíntesis (Tabla 2) y que por su contenido de nutrientes podría generar un buen crecimiento de $C$. sorokiniana tal como lo haría un fertilizante inorgánico.

En el test de crecimiento de $C$. sorokiniana en medios inorgánicos y el lixiviado de gallinaza se encontró que el medio inorgánico Guillard permitió obtener la mayor densidad celular de la microalga con $130 \pm 17,3 \times 10^{7}$ células $/ \mathrm{ml}$ con un tiempo de generación ( $\mathrm{g}$ ) de 1,5 días y una velocidad específica de crecimiento de 0,7 generaciones/día. Al comparar los tiempos de generación para los medios de cultivo se encontró que fueron similares entre sí exceptuando al medio Sueoka (Tabla 3). Los datos indican que el crecimiento en lixiviado de gallinaza presenta una fase de latencia más prolongada que en los medios inorgánicos (Tabla 3), lo cual se debe a adaptaciones fisiológicas de la microalga para la incorporación de los compuestos orgánicos presentes en el medio (Xu et al., 2006; Chinnasamy et al., 2010).

En los medios de cultivo con lixiviado de gallinaza la densidad celular máxima fue de $33 \pm 6,7 \times 10^{7}$ células/ $\mathrm{ml}$ en la concentración del $30 \%$, al comparar estos resultados con los obtenidos por Rosales et al (2007) para una concentración del $36 \%$ de lixiviado de gallinaza (densidad celular de 22,8 $\pm 0,04 \times 10^{7}$ células $/ \mathrm{ml}$, velocidad de crecimiento de 0,35 generaciones/día y un tiempo de generación de 1,97 días), se encuentra que en el presente trabajo se logró una alta densidad celular, alta velocidad de crecimiento y un menor tiempo de generación (g), con una menor concentración de lixiviado de gallinaza (Tabla 3). Esto puede deberse a diferencias en el contenido de nutrientes del lixiviado de gallinaza, ya que los estiércoles líquidos poseen una alta heterogeneidad dependiendo de su origen (Cheeke et al., 1977; Groeneweg y Schluter, 1981; Travieso et al., 2006).

En el experimento en que se comparo los mejores medios de cultivo para C. sorokiniana con el medio Remital reportado por Muñoz (2009), se encontró que el lixiviado de gallinaza no permitió un incremento de la densidad celular de $C$. sorokiniana comparable a los medios inorgánicos Remital y Guillard (Tabla 4). Esto puede estar relacionado a la calidad de nutrientes aportada por los medios de cultivo, Moronta et al., (2006) reportan que en condiciones axénicas, C. sorokiniana tuvo una densidad celular en autotrofia de $1,8 \times 10^{7}$ células $/ \mathrm{ml}$ mientras que en régimen mixotrófico se obtuvo $1,2 \times 10^{8}$ células $/ \mathrm{ml}$. Es decir, se obtuvo un mayor crecimiento celular en mixotrofia, contrario a lo observado en el presente trabajo donde las máxi- mas densidades celulares de $C$. sorokiniana se lograron en los medios de cultivo con régimen autotrófico $\left(8,6 \pm 0,7 \times 10^{8}\right.$ células $\left./ \mathrm{ml}\right)$, datos similares también son reportados por Rosales et al., (2007) (autotrófico: $1,3 \times 10^{8}$ células $/ \mathrm{ml}$; mixotrofico: $2,3 \times 10^{8}$ células $/ \mathrm{ml}$ ). Esto puede estar relacionado con la disponibilidad y calidad de nutrientes empleados en la mixotrofia y en la autotrofia, es decir si en el medio de cultivo mixotrófico se emplean sustancias fácilmente metabolizables y en el autotrófico bajos niveles de nitrógeno y fósforo, en consecuencia el crecimiento de $C$. sorokiniana se verá favorecido en el medio de cultivo mixotrófico (Xu et al., 2006; Kumar et al., 2010) (Tabla 4).

En conclusión, los datos obtenidos indican que para la producción de biomasa de $C$. sorokiniana se debe emplear como fuente de nutrientes el abono Remital$M$ en régimen autotrófico, lo cual es congruente con lo reportado por Muñoz (2009).

Al comparar el crecimiento de $C$. sorokiniana en concentraciones de Remital: 1.0, 2.0, 3.0, 4.0 y 5.0 g/L, se encontró que a mayor concentración del fertilizante se presentaba una densidad celular mayor de la microalga, pero el análisis estadístico (ANOVA) no mostró diferencias significativas entre los tratamientos, con un valor de $\mathrm{P}=0,123$ (Figura 3 y Tabla 5). Por tanto incrementar la concentración de Remital no aumentó significativamente la densidad celular de $C$. sorokiniana, de tal manera que para producir biomasa microalgal se puede usar concentraciones bajas de fertilizante como $1 \mathrm{~g} / \mathrm{L}$, y así reducir los costos de producción haciendo viable el uso biotecnológico de $C$. sorokiniana.

\section{Conclusion}

El medio de cultivo Remital, presentó las mejores condiciones para el crecimiento de la microalga $C$. sorokiniana, comparado con los medios Guillard, Sueoka (condición autotrófica) y lixiviado de gallinaza (condición mixotrófica). El medio Remital permite un rápido crecimiento celular, en bajas concentraciones $(1 \mathrm{~g} / \mathrm{L})$. Por tanto puede ser promisorio para la producción masiva de biomasa de $C$. sorokiniana con fines industriales.

\section{Agradecimientos}

Al Instituto de Investigaciones de la Orinoquía Colombiana-IIOC, quien financió este trabajo, al apoyo técnico del Grupo GRITOX del Instituto de Acuicultura de los Llanos-IALL, del Laboratorio de Biología, Nutrición Animal y del Laboratorio de Aguas de la Universidad de los Llanos. 


\section{Referencias}

Benedetti A, Canalli S, Lianello F. 1998. La fertilizzazione organica del suoli, En: Sequi, P. (ed.). Fertilizzanti Organici. Edizione L'Informatore Agrario, Roma.

Camargo J, Alonso A. Ecological and toxicological effects of inorganic nitrogen pollution in aquatic ecosystems: A global assessment. Environment International. 2006; 32(6): 831-849.

Cheeke P, Gasper E, Boersma L, Oldfield J. Nutritional evaluation with rats of algae (Chlorella) grown on swine manure. Nutrition Reports International. 1977; 16:579-585.

Chinnasamy S, Bhatnagar A, Hunt RW, Das KC., Microalgae cultivation in a wastewater dominated by carpet mill effluents for biofuel applications, Bioresource Technology 2010; 101: 3097-3105.

Chisti Y. Biodiesel from microalgae. Biotechnology Advances, 2007; 25: 294-306.

Costa R, Medri W, Perdomo C. High-rate pond for treatment of piggery wastes. Water Science and Technology. 2000; 42: 357362.

Fernández J, Benito J. 2009, Revalorización de subproductos en el sector de producción de huevos, Tratamientos de revalorización de gallinaza líquida, Asociación Española de Productores de Huevos, Madrid.

Groeneweg J, Schluter M. Mass production of fresh water rotifers on liquid wastes. II. Mass production of Brachionus rubens in the effluent of high-rate algal ponds used for the the treatment of piggery waste. Aquaculture. 1981; 25:25-33.

Hernández J, Cruz A. 1993, Boletín informativo sobre el uso de subproductos: Gallinaza. Ministerio de Agricultura y Ganadería. San José de Costa Rica.

ICONTEC. 1994, Manual de métodos analíticos para el control de calidad del agua-GTC 2, Publicaciones ICONTEC, Bogotá.

ICONTEC. 1999a, Alimentos para animales, preparación para las muestras de ensayo-NTC 4647, Publicaciones ICONTEC, Bogotá.

ICONTEC. 1999b, Alimentos para animales, determinación del contenido de nitrógeno y cálculo de proteína cruda. Método Kjeldahl, Publicaciones ICONTEC, Bogotá.

ICONTEC. 2001, Alimentos para animales, determinación del contenido de grasa, Publicaciones ICONTEC, Bogotá.

ICONTEC. 2002, Alimentos para animales, determinación del contenido fibra cruda. Método con filtrado intermedio, Publicaciones ICONTEC, Bogotá.

ICONTEC. 2004, Productos para la industria agrícola. Productos orgánicos usados como abonos y fertilizantes y enmiendas de suelo, Publicaciones ICONTEC, Bogotá.

ICONTEC. 2006, Alimentos para animales, determinación de ceniza cruda-NTC 4648, Publicaciones ICONTEC, Bogotá.

IBVF, 2011. Medios de cultivo para microalgas. Disponible on line en: http://www.ibvf.csic.es/colecciones_cultivo
Jiménez-Pérez M, Sánchez-Castillo P, Romera O, Fernández-Moreno $D$, Pérez-Martínez $C$. Growth and nutrient removal in free and immobilized planctonic green algae isolated from pig manure. Enzyme and Microbiology Technology. 2004; 34:392-398.

Kebede-Westhead E, Pizarro C, Mulbry W. Treatment of dairy manure effluent using freshwater algae: Elemental composition of algal biomass at different manure loading rates. Journal of Agronomy and Food Chemistry, 2004; 52:7293-7296.

Knud-Hansen C. 1998, Pond fertilization: Ecological approach and practical application. Pond dynamics/aquaculture collaborative research support program. State University of Oregon. Oregon.

Kumar M, Miao Z, Wyatt S. Influence of nutrient loads, feeding frequency and inoculums source on growth of Chlorella vulgaris in digested piggery effluent culture medium. Bioresource Technology. 2010; 101 (15):6012-6018.

Lipstein B, Hurwitz S. The nutritional value of algae for poultry. Dried Chlorella in broiler diets. British Poultry Science. 1980; 21:9-21.

Lobban C, Chapman D, Kremer B. 1988, Experimental phycology: A laboratory manual. Cambridge University Press, New York.

Lu L, Wang X, Xu M. Effect of Zinc and composting time on dynamics of different soluble copper in chicken manures. Agricultural sciences in China. 2010; 9(6): 861-870.

Moronta R, Mora R, Morales E. Respuesta de la microalga ChloreIla sorokiniana al $\mathrm{pH}$, salinidad y temperatura en condiciones axénicas y no axénicas. Revista de la Facultad de Agronomía. 2006; 23 (1): 27-41.

Muñoz M. 2009, Efecto de diferentes medios de cultivo sobre el crecimiento y el contenido proteico de Chlorella vulgaris. Trabajo de Grado, Universidad Jorge Tadeo Lozano, Bogotá.

Olguín E, Galicia S, Angulo-Guerrero O, Hernández E. The effect of low light flux and nitrogen deficiency on the chemical composition of Spirulina sp. (Arthrospira) grown on digested pig waste. Bioresource technology. 2001;77:19-24.

Pizarro C, Kebede-Westhead E, Mulbry W. Nitrogen and phosphorus removal rates using small algal turfs grown with dairy manure. Journal of Applied Phycology. 2002; 14:469-473.

Rosales N, Bermúdez J, Moronta R, Morales E. Gallinaza: un residual avícola como fuente alternative de nutrientes para producción de biomasa microalgal. Revista Colombiana de Biotecnología. 2007;9(1):41-48.

Sevrin-Reyssac J. Biotreatment of swine manure by production of aquatic valuable biomasses. Agriculture, Ecosystem and Environment. 1998; 68:177-186.

Singh S, Goyal D. Microbial and plant derived biomass for removal of heavy metals from wastewater. Bioresources Technology. 2007; 98:2343-2357.

Spoehr H, Milner $\mathrm{H}$. The chemical composition of Chlorella. Effect of the environmental conditions. Plant Physiology. 1949; 24:120-149. 
Spolaore P, Joannis-Cassan C, Duran E, Isambert A. Commercial applications of microalgae. Journal of Biosciences and Bioengineering. 2006; 101: 87-96.

Travieso L, Benitez F, Sanchez E, Borja R, Martín A, Colmenarejo M. Batch mixed culture of Chlorella vulgaris using settled and diluted piggery waste. Ecological Engineering. 2006;28 (2): 158-165.

Ugwu C, Ogbonna J, Takana H. Characterization of light utilization and biomass yields of Chlorella sorokiniana in inclined outdoor tubular photobioreactors equipped with static mixers. Proceeds on Biochemistry. 2005; 40:3406-3411.

Vasquez J. Los residuos orgánicos y la actividad agropecuaria. Vida rural. 1994;2:82-84.
Venglovsky J, Martinez J, Placha I. Hygienic and ecological risks connected with utilization of animal manures and biosolids in agriculture. Livestock Science. 2006; 102(3):197-203.

Wang L, Li Y, Chen P, Min M, Chen Y, Zhu J, Ruan R. Anaerobic digested dairy manure as a nutrient supplement for cultivation of oil-rich green microalgae Chlorella sp. Bioresource Technology. 2010; 101(8):2623-2628.

Wehr J, Sheath R. 2003, Freshwater algae of North America: Ecology and classification, Aquatic Ecology Series, Academic Press, San Diego.

Xu H, Miao X, Wu Q. High quality biodiesel production from a microalga Chlorella protothecoides by heterotrophic growth in fermenters. Journal of Biotechnology. 2006; 126: 499-507. 